$3.41 \mathrm{~ms}$ in the control group) according to our data. The ambulatory arterial stiffness index (AASI) was $0.88 \pm 0.02$, which was exceeded the control group data $(0.55 \pm 0.01)$. The increase in diameter of the artery(reactive hyperemia test using) in the study group was less than 10\% (8.59 $\pm 0.36 \%)$. Changes of ECO-indicators: reduction of peak speed of wave $\mathrm{VE}$; increase of peak speed of wave VA; reduction of the VE/ VA ratio; prolongation of the isovolumic relaxation phase; an increase in delayed early diastolic filling may be considered as initial manifestations of diastolic dysfunction in the examined children.

Conclusion The ambulatory arterial stiffness index was $0.88 \pm$ 0.02 in the study groupe. Increase the diameter of the artery after reactive hyperemia test in the study group was less than $10 \%$, KIM - 0,56 $\pm 0,03 \mathrm{~mm}$ ). The use of integral morfofunctional parameters provides an objective criterium to optimize diagnosis, for the purpose of risk evaluation in the children with $\mathrm{AH}$ and allows controlling the development of cardiovascular pathology

\section{GP32 ECHOCARDIOGRAPHIC FOLLOW-UP OF CHILDREN WITH SUBAORTIC STENOSIS}

${ }^{1}$ Betül Çınar*, Sezen Ugan Atik ${ }^{2},{ }^{2}$ Ayş̧e Güler Eroğlu, ${ }^{2}$ Levent Saltık. ${ }^{1}$ Istanbul University Cerrahpaşa Medical Faculty, Department of Pediatrics, istanbul, Turkey; ${ }^{2}$ Istanbul University Cerrahpaşa Medical Faculty, Department of Pediatric Cardiology, istanbul, Turkey

\subsection{6/archdischild-2019-epa.98}

Introduction Subaortic stenosis(SAS) accounts for $8-30 \%$ of patients with congenital left ventricular outflow tract obstructions. Allthough progressive SAS occurs in many patients, the exact etiology and factors contributing to progression remains unknown. In this study, we evaluated the natural course of SAS, associated aortic regurgitation (AR), the factors affecting the progression of SAS and AR and the outcomes of surgery during the long-term follow-up with echocardiography.

Materials and Methods The study included 105 patients who were evaluated and followed with echocardiography at our institution between 1990 and 2017 with SAS, consisting of either a thin ridge or a thicker but discrete obstruction with a muscular base. Patients with incomplete medical records, abnormal ventricular function, and lesions other than AR were excluded. The last examination prior to any surgical intervention provided our final measurements. The level of narrowing of the LVOT, the distance between right coronary cusp and ridge and the anulus of the aortic valve were determined with two-dimensional echocardiography. Continuous-wave Doppler was used through an apical five-chamber view to record the maximum peak and mean systolic instantaneous gradient across the supravalvular narrowing. A multivariate analysis with Cox proportional hazards modeling was performed to adjust for the different distributions of variables between groups. The enter method was used in logistic regression analysis.

Results Among 105 patients (median, 5 years at initial echocardiography), 64\% were male and $36 \%$ were female. The patients were followed median 6.6 years. Aortic valve morphology was tricuspit in $95.2 \%$ and bicuspid in $4.8 \%$. The median distance of discret membran from the right coronary cusp was $6.4 \mathrm{~mm}$. The degree of SAS staid the same in $60 \%$, progressed in $29 \%$ and $11 \%$ underwent surgery after initial echocardiography. AR did not develope in $21(20 \%)$, not deteriorate in $41(39 \%)$ and progressed in $43(41 \%)$ patients.
Surgery was performed in $38(63.8 \%)$ patients. $6(5.7 \%)$ patients underwent reoperation.

Conclussion We recommend careful and frequent evaluation for patients with moderate stenosis because surgery may be needed depending on the severity of stenosis and AR. Postoperatively, follow-up is required.

\section{GP33 HIGH PREVALENCE NOONAN SYNDROME IN RUSSIAN CHILDREN WITH HYPERTROPHIC CARDIOMYOPATHY, DIAGNOSED BY NEXT GENERATION SEQUENCING}

${ }^{1} \mathrm{~N}$ Zhurkova $\mathrm{V}^{*},{ }^{1} \mathrm{~K}$ Savostyanov $\mathrm{V},{ }^{1} \mathrm{AA}$ Pushkov, ${ }^{2} \mathrm{ND}$ Vashakmadze, ${ }^{1} \mathrm{~N}$ Fedorova $\mathrm{V}$, ${ }^{1} \mathrm{OP}$ Zharova, ${ }^{1} \mathrm{LA}$ Gandaeva, ${ }^{1} \mathrm{NA}$ Sdvigova, ${ }^{3} \mathrm{OB}$ Kondakova, ${ }^{1} \mathrm{EN}$ Basargina. ${ }^{1}$ National Medical Research Center of Children's Health of the Ministry of Health of the Russian Federation, Moscow, Russian Federation; ${ }^{2}$ Pirogov Russian National Research Medical University, Moscow, Russian Federation; ${ }^{3}$ Scientific and Practical Center of Pediatric psychoneurology Department of Health of Moscow, Moscow, Russian Federation

\subsection{6/archdischild-2019-epa.99}

Backgraund Noonan syndrome - is rare autosomal dominant disorder from RASopathies group, characterized by facial dysmorphism, short stature, hypertrophic cardiomyopathy, congenital heart defects.

Patients and Methods We have examined 47 patients with hypertrophic cardiomyopathy aged 1 to 17 years. Target areas of the exome were investigated by NGS. Bioinformatic analysis was carried out using the Alamut software. Validation of the identified variants was carried out by the Sanger method.

Results The diagnosis was confirmed in 13 patients with hypertrophic cardiomyopathy and cardiologic abnormalities. Short stature and facial features have all our patients. Congenital heart defect, including pulmonic stenosis diagnosed in 6 cases.

RAF1 mutations were identified in 7 patients, PTPN11 - in 3 patients, SOS1- in 1 patient, SOS 2 - in 1 patient, and RIT1 - in 1 patient. Most frequent RAF1 mutation was c.770C $>T$, p. S257L (5 from 7 cases). Girl with mutation in RIT-1 was with phenotype Noonan syndrome, but she also have left ventricular noncompaction and skin cafe-au-lait spots.

Conclusion Noonan syndrome was diagnosed in 28\% hypertrophic cardiomyopathy patients.

Mutation c.770C $>$ T, p.S257L in RAF1 gen is most common in hypertrophic cardiomyopathy patients with Noonan syndrome

\section{GP34 QUALITY OF LIFE IN CARDIOPATHIC CHILDREN AND ADOLESCENTS}

Giulia Amodeo*, Teresa Grimaldi Capitello. Ospedale Pediatrco Bambino gesù, Roma, Italy

\subsection{6/archdischild-2019-epa.100}

Introduction The concept of quality of life is broad and multidimensional. The temporal dimension of its evaluation is very important because the quality of life is not a stable and defined condition, but changes over time.

Heart disease is classified among the most severe chronic disabilities in children and as more and more children and adolescents with severe cardiac defects survive into adulthood, the quality of their life becomes increasingly important.

The quality of life in cardiopaths may vary depending on various factors, ranging from the intake of a drug therapy, to 